\title{
Handcrafted and Deep Learning-Based Radiomic Models Can Distinguish GBM from Brain Metastasis
}

\author{
Zhiyuan Liu $\mathbb{D}^{1,2}$ Zekun Jiang $\mathbb{D}^{3},{ }^{3}$ Li Meng $\mathbb{D}^{2,4}$ Jun Yang $\mathbb{D}^{5},{ }^{5}$ Ying Liu $\mathbb{D}^{6},{ }^{6}$ \\ Yingying Zhang $\mathbb{D}^{1,2}$ Haiqin Peng $\mathbb{D}^{1,2}$ Jiahui Li $\mathbb{D}^{1,2}$ Gang Xiao $\mathbb{D}^{1,2}$ Zijian Zhang $\mathbb{D}^{1,2}$ \\ and Rongrong Zhou $\mathbb{D}^{1,2}$ \\ ${ }^{1}$ Department of Oncology, Xiangya Hospital, Central South University, Changsha 410008, China \\ ${ }^{2}$ Xiangya Lung Cancer Center, Xiangya Hospital, Central South University, Changsha 410008, China \\ ${ }^{3}$ Shandong Key Laboratory of Medical Physics and Image Processing, \\ Shandong Institute of Industrial Technology for Health Sciences and Precision Medicine, School of Physics and Electronics, \\ Shandong Normal University, Jinan 250358, Shandong, China \\ ${ }^{4}$ Department of Radiology, Xiangya Hospital, Central South University, Changsha 410008, China \\ ${ }^{5}$ Department of Radiology, The Third Affiliated Hospital of Kunming Medical University, Yunnan Cancer Hospita and Center, \\ Kunming 650100, Yunnan, China \\ ${ }^{6}$ Department of Radiation Oncology, Nanfang Hospital, Southern Medical University, Guangzhou 510515, China
}

Correspondence should be addressed to Zijian Zhang; wanzzj@csu.edu.cn and Rongrong Zhou; 664990095@qq.com

Received 26 February 2021; Revised 22 April 2021; Accepted 24 May 2021; Published 4 June 2021

Academic Editor: Jia Cheng Lou

Copyright (c) 2021 Zhiyuan Liu et al. This is an open access article distributed under the Creative Commons Attribution License, which permits unrestricted use, distribution, and reproduction in any medium, provided the original work is properly cited.

\begin{abstract}
Objective. The purpose of this study was to investigate the feasibility of applying handcrafted radiomics (HCR) and deep learningbased radiomics (DLR) for the accurate preoperative classification of glioblastoma (GBM) and solitary brain metastasis (BM). Methods. A retrospective analysis of the magnetic resonance imaging (MRI) data of 140 patients (110 in the training dataset and 30 in the test dataset) with GBM and 128 patients (98 in the training dataset and 30 in the test dataset) with BM confirmed by surgical pathology was performed. The regions of interest (ROIs) on T1-weighted imaging (T1WI), T2-weighted imaging (T2WI), and contrast-enhanced T1WI (T1CE) were drawn manually, and then, HCR and DLR analyses were performed. On this basis, different machine learning algorithms were implemented and compared to find the optimal modeling method. The final classifiers were identified and validated for different MRI modalities using HCR features and HCR + DLR features. By analyzing the receiver operating characteristic (ROC) curve, the area under the curve (AUC), accuracy, sensitivity, and specificity were calculated to evaluate the predictive efficacy of different methods. Results. In multiclassifier modeling, random forest modeling showed the best distinguishing performance among all MRI modalities. HCR models already showed good results for distinguishing between the two types of brain tumors in the test dataset (T1WI, AUC $=0.86$; T2WI, AUC $=0.76$; T1CE, AUC $=0.93$ ). By adding DLR features, all AUCs showed significant improvement (T1WI, AUC $=0.87$; T2WI, AUC $=0.80$; T1CE, AUC $=0.97 ; p<0.05$ ). The T1CE-based radiomic model showed the best classification performance (AUC $=0.99$ in the training dataset and AUC $=0.97$ in the test dataset), surpassing the other MRI modalities $(p<0.05)$. The multimodality radiomic model also showed robust performance $(\mathrm{AUC}=1$ in the training dataset and $\mathrm{AUC}=0.84$ in the test dataset). Conclusion. Machine learning models using MRI radiomic features can help distinguish GBM from BM effectively, especially the combination of HCR and DLR features.
\end{abstract}

\section{Introduction}

Brain metastases (BMs) are the most common tumors of the central nervous system (CNS), with an incidence of approximately $7-14$ per 100,000 population [1], whereas glioblastoma (GBM) has an incidence rate of 3.22 per 100,000 population and is the most common malignant primary brain tumor in adults [2]. According to the World Health Organization (WHO) classification of CNS tumors, gliomas can be categorized as grades I-IV based on 
histological characteristics; GBM is categorized as a WHO grade IV glioma, accounting for the majority of gliomas [3]. According to the statistics of the Central Brain Tumor Registry of the United States (CBTRUS), the incidence of BM and GBM increases each year [2]. Patients with $B M$ have a median survival of 4-6 months [4], while the median survival of GBM patients is 14 months [5], despite surgery, chemotherapy, and radiation therapy. At present, the standard treatment for BM is stereotactic radiotherapy, while the most effective therapeutic method for GBM is surgery. Therefore, an accurate preoperative diagnosis is of significance for surgical planning, determining the extent of resection [6], evaluating the need for neoadjuvant therapy, defining the radiation therapy field, and counseling patients and their families [7].

It is generally accepted that magnetic resonance imaging (MRI) is an important modality for evaluating brain tumors. In patients with a history of systemic cancer and multiple lesions, the differentiation of BM from GBM may be easily achieved using conventional MRI. However, single metastases were estimated to occur in more than $25 \%$ of cases of BM [8]. Additionally, systemic malignancy was present in approximately $3 \%$ of high-grade glioma cases [9], and multifocal lesions accounted for up to $20 \%$ of GBM cases in some reports [10]. Furthermore, as both BM and GBM can present with contrast-enhancing and necrotic areas, they often present a similar anatomical appearance on MRI [8]. Therefore, the use of conventional MRI in differentiating between single BMs and GBM lesions is limited.

Generally, BM and GBM are distinguished by some observable MRI features. Lesions that show nodular or ring-shaped inhomogeneous contrast enhancement and hypointense signals on T1-weighted imaging (T1WI) and hyperintense signals on T2-weighted imaging (T2WI) are visible on conventional MRI for both diseases. Compared with GBM lesions, most BM lesions are multifocal, have relatively clear boundaries, and are surrounded by severe edema. Moreover, BM lesions have smaller volumes, smaller areas of necrosis and cystic degeneration, and less enhancement than GBM lesions. Nevertheless, these methods, with limited sensitivity and specificity, are susceptible to individual subjectivity, resulting in differences among radiologists. However, the use of multiple MRI techniques is expected to significantly improve the diagnostic results. Several studies have differentiated between GBM and BM based on multiparametric MRI data, including data from advanced imaging methods such as diffusion $[11,12]$, perfusion $[13,14]$, and MR spectroscopy $[15,16]$. Askaner et al. made a differential diagnosis by statistically analyzing the relative cerebral blood volume of the solid tumor area, peritumor area, and adjacent area on perfusion-weighted imaging (PWI) [17]. The apparent diffusion coefficient was used to compare and distinguish both diseases on diffusion-weighted imaging (DWI) by Lee et al. [18]. Nevertheless, there is no widely held standard to distinguish between GBM and BM, except histopathological evaluation. Moreover, these advanced imaging methods are not readymade tools in most radiology departments because they are relatively time consuming, which limits their clinical promotion.

While histopathological evaluation is currently the gold standard for brain tumor diagnosis [19], there is a growing body of evidence that the combination of quantitative imaging and machine learning algorithms can help with the noninvasive differentiation of brain neoplasms based on pretreatment MRI [20]. Radiomics is an emerging field that aims to utilize the full potential of medical imaging by extracting a large number of quantitative features, including tumor intensity, shape, and texture [21]. Radiomics has recently emerged as a powerful methodology to quantify the characteristics of tumors and mine more biological information in a noninvasive manner [22]. Many studies have demonstrated that distinct tumor types in many organs can be quantified by radiomic analysis, and the results of radiomics can be used as imaging biomarkers to support clinical decision making [23-25]. Radiomics can also reveal novel characteristics of brain tumors, as demonstrated by a recent study. Radiomic analysis has been shown to improve diagnosis, prognosis, and decision making in the treatment of patients over standard radiological assessment [26]. Machine learning models can combine a large number of variables of different data types in a single model, thereby maximizing the efficacy of prediction testing. Machine learning technology has been widely used to diagnose various types of tumors.

Previous studies have shown that radiomics offers important advantages in the assessment of the underlying tumor pathophysiology and improves the ability to distinguish between tumors [27-29]. Although multiple classifiers have been found in many previous radiomic studies, the main purpose was to find the best classifier and not validate the additional radiomic features. Because different radiomic feature groups may have their own advantages or disadvantages due to the feature extraction method, we hypothesized that the combined use of handcrafted radiomics (HCR) and deep learning-based radiomics (DLR) might provide extra benefits. To our knowledge, there have been no reports validating the potential of the combination of HCR and DLR for the classification of GBM and solitary BM lesions. Thus, the present study sought to differentiate single BM from GBM lesions by combining high-dimensional radiomic features based on conventional MRI and machine learning technologies.

\section{Materials and Methods}

The study was approved by the Institutional Review Board (IRB) of Xiangya Hospital. According to the relevant guidelines and regulations of the retrospective study, the requirement for informed consent was waived. The study workflow overview is shown in Figure 1.

2.1. Patient Data. This was a retrospective analysis of 268 patients with GBM and BM from Xiangya Hospital, Yunnan Cancer Hospital, and Nanfang Hospital, proven pathologically between January 1, 2010, and December 31, 2018. Preoperative routine plain and enhanced MRI scans were required for patients to be eligible for inclusion in the study. Patients who had a treatment history (including surgery and radiotherapy) related to GBM or BM were excluded. The 268 

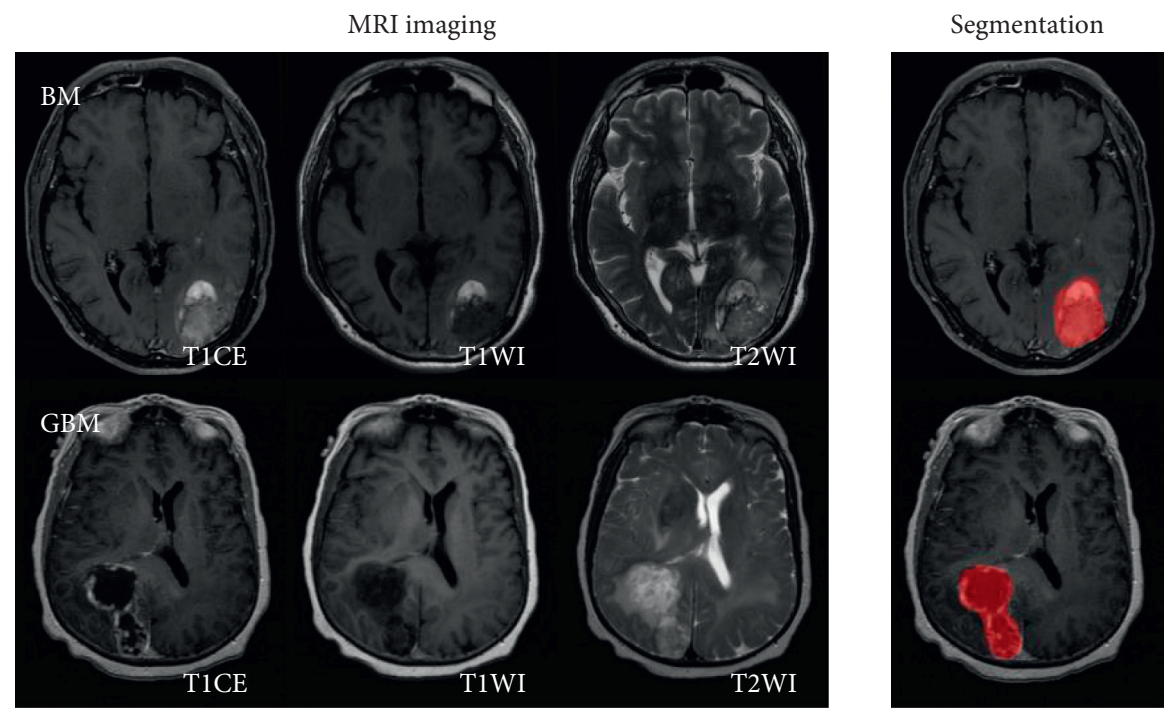

Radiomics analysis
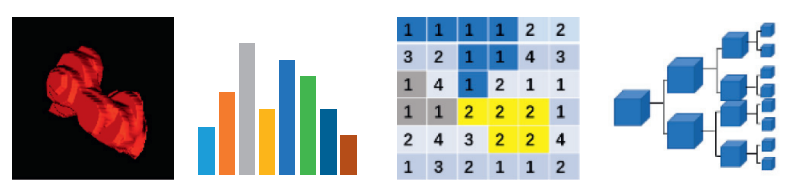

HCR features

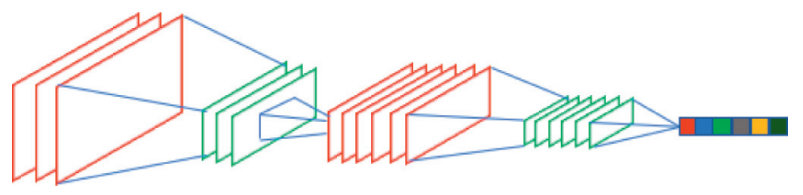

DLR features

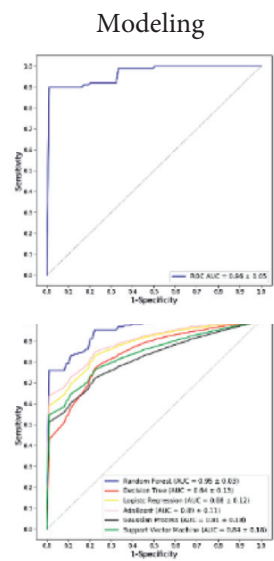

FIgURE 1: Study workflow overview.

patients were separated into two groups: 208 (98 BM and 110 GBM) patients from Xiangya Hospital were included in the training dataset and 60 (30 BM and $30 \mathrm{GBM}$ ) patients from Yunnan Cancer Hospital and Nanfang Hospital were included in the test dataset. The diagnoses of all patients were confirmed histologically on the basis of the $2016 \mathrm{WHO}$ classification system.

2.2. MRI Protocol. All MRI examinations were conducted in the radiology department of Xiangya Hospital, Yunnan Cancer Hospital, and Nanfang Hospital with a 3.0-T MRI system. High-quality MRI images were obtained using the following protocols: axial T1WI: layer thickness $=5 \mathrm{~mm}$, layer spacing $=1.5 \mathrm{~mm}$, matrix $=320 \times 256$, and field of view $($ FOV $)=24 \times 24 \mathrm{~cm}$; axial T2WI: layer thickness $=5 \mathrm{~mm}$, layer spacing $=1.5 \mathrm{~mm}$, matrix $=384 \times$ 384 , and $\mathrm{FOV}=24 \times 24 \mathrm{~cm}$; and axial contrast-enhanced T1WI (T1CE): layer thickness $=5 \mathrm{~mm}$, layer spacing $=1.5 \mathrm{~mm}$, matrix $=320 \times 256$, and FOV $=24 \times 24 \mathrm{~cm}$. All MRI images were retrieved from the picture archiving and communication system for further image feature extraction.
2.3. Region of Interest (ROI) Preprocessing. Through noise reduction, offset field correction, and strict object internal registration, we preprocessed each image using the public software package FSL. Histogram matching was performed such that the intensity levels between various objects were comparable. All images were assessed by two neuroradiologists (each with 5-10 years of work experience) independently. The ROIs of the entire tumor on T1WI, T2WI, and T1CE images were created manually around the enhanced part of the tumor layer by layer using ITK-SNAP software [30]; areas of macroscopic necrosis, cystic changes, and edema were avoided. A third senior neuroradiologist (with 15 years of work experience) reexamined the images and made a final diagnosis when there was an inconsistency between the two neuroradiologists.

2.4. HCR Feature Extraction. Based on the segmentation of ROIs, we performed HCR feature extraction using PyRadiomics [31]. The high-throughput HCR features included 16 shape-based features, 18 histogram features, 20 gray level co-occurrence matrix (GLCM) features, 14 gray level dependence matrix (GLDM) features, 16 gray level run length 
matrix (GLRLM) features, 16 gray level size zone matrix (GLSZM) features, 5 neighboring gray tone difference matrix (NGTDM) features, 728 wavelet features, and 273 Laplacian of Gaussian (LoG) features. The details of these features are available in PyRadiomics documentation (https://pyradiomics.readthedocs.io/en/latest/index.html). Finally, 1106 HCR features were extracted from each MRI ROI, and 3318 HCR features were extracted from each patient.

2.5. DLR Feature Extraction. A convolutional neural network $(\mathrm{CNN})$ was also used to extract and summarize MRI features. Here, we used a pretrained ResNet transferred from ImageNet, which was built by Keras. The last fully convolutional layer was reset, and each ResNet could extract 1000 features from each MRI modality.

2.6. Feature Selection and Machine Learning Modeling. Based on the HCR and DLR features, the random forest(RF-) based Boruta algorithm was applied to select the optimal feature groups. Boruta is a wrapper algorithm that performs robust, statistically grounded feature selection for all relevant features [32]. By comparing the importance of the original attribute with the importance of its randomized copies [33], all relevant features were selected for subsequent modeling. One RF-Boruta selection example is shown in Figure 2. Then, for the selected HCR features and HCR + DLR features of the three MRI modalities, a total of 6 radiomic feature groups were advanced to the next modeling step. To find the optimal modeling method, six different machine learning algorithms were adopted to build the classifiers for the 6 feature groups. RF, decision tree, logistic regression, AdaBoost, Gaussian processing, and support vector machine were implemented using 10 -fold crossvalidation of the training dataset in turn. Receiver operating characteristic (ROC) curve analysis was performed, and the area under the curve (AUC) of each model was obtained to evaluate the predictive performance.

2.7. Model Establishment and Validation. After finding the best modeling method, each classifier was established and validated in the training dataset and tested in the test dataset. The HCR models of the T1CE, T1WI, T2WI, and multimodality features were built and compared; then, DLR features were added, and the above process was repeated. Through comparison of the ROC curves and AUC values, the optimal MRI modality and machine learning model were identified.

2.8. Statistical Analysis. All machine learning algorithms were implemented using scikit-learn and Keras libraries, and statistical analysis was carried out using SPSS (version 26, IBM Corporation, Armonk, NY, USA). Independent $t$-tests were used to assess differences in continuous variables between the patient groups, and Fisher's exact tests were used to assess noncontinuous variables. The DeLong test was

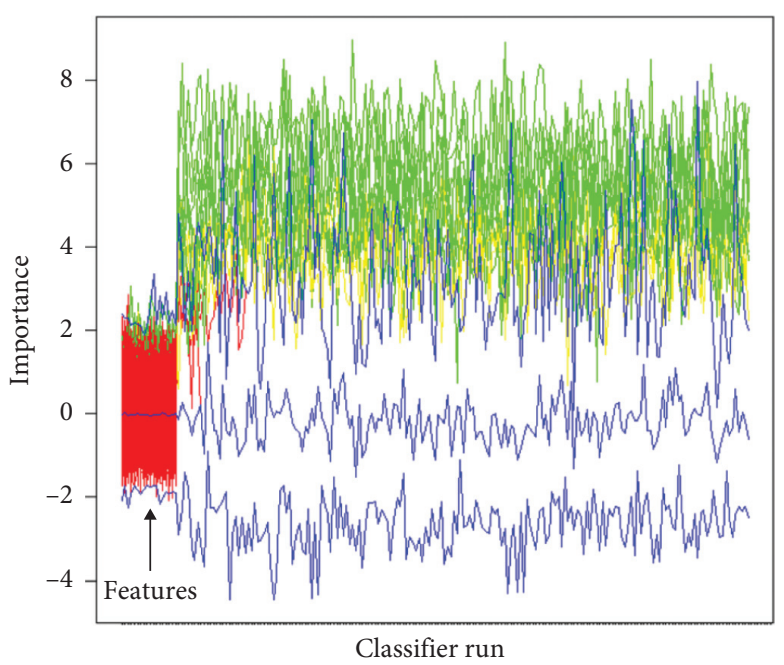

FIgure 2: The Boruta selection of HCR + DLR features of T1CE data. Green and yellow indicate high-importance features, and blue and red indicate low-importance features.

performed to compare ROC curves. A $P$ value $<0.05$ was considered statistically significant.

\section{Results}

3.1. Patient Characteristics. The clinical characteristics of the patients in the training and test cohorts are given in Table 1. There was no significant difference in age or sex between patients with GBM and BM in the two cohorts.

\subsection{Radiomics Features and Machine Learning Modeling.} A total of 1106 HCR features and 1000 DLR features were extracted for each case from T1WI, T2WI, and T1CE data. By Boruta selection, the relevant features were selected; the details of each feature group are given in Supplementary Material 1. For all 6 feature groups, the RF models all showed better performance than the other machine learning algorithms $(p<0.05)$ (Figure 3).

With only HCR features, T1CE showed the best distinguishing performance ( $\mathrm{AUC}=0.99$, in the training dataset; AUC $=0.93$, in the test dataset), which was significantly better than that of T1WI and T2WI $(p<0.05)$. Adding DLR features, T1CE showed significant improvement $(\mathrm{AUC}=0.99$, in the training dataset; $\mathrm{AUC}=0.97$, in the test dataset; $p<0.05$ ) and remained the best model. T1WI (AUC $=0.99$, in the training dataset; $\mathrm{AUC}=0.87$, in the test dataset; $p<0.05)$ and T2WI (AUC $=1.00$, in the training dataset; $\mathrm{AUC}=0.80$, in the test dataset; $p<0.05$ ) also showed significant improvement. All details are shown in Figure 4. Supplementary Material 2 shows the robust performance in the training dataset using 10 -fold cross-validation.

Generally, multimodality radiomic models show better diagnostic performance than single-modality models. In our study, the multimodality model showed an AUC of 1.00 in the training dataset and an AUC of 0.84 in the test dataset, which were lower than those of the T1CE- and T1WI-based radiomic models $(p<0.05)$. Table 2 and Figure 5 show a 
TABLE 1: Cohort characteristics of 268 patients with BM and GBM.

\begin{tabular}{|c|c|c|c|c|c|}
\hline Characteristics & Groups & Full $(n=268)$ & Training $(n=208)$ & Testing $(n=60)$ & $P$ value \\
\hline \multirow{2}{*}{ Age (years) } & Mean \pm & 55.48 & 55.63 & 54.96 & \multirow{2}{*}{0.74} \\
\hline & SD & 9.18 & 9.36 & 8.68 & \\
\hline \multirow{2}{*}{ Gender } & Male & 170 & 138 & 33 & \multirow{2}{*}{0.22} \\
\hline & Female & 98 & 70 & 27 & \\
\hline \multirow{2}{*}{ Tumor types } & $\mathrm{BM}$ & 128 & 98 & 30 & \\
\hline & GBM & 140 & 110 & 30 & \\
\hline
\end{tabular}

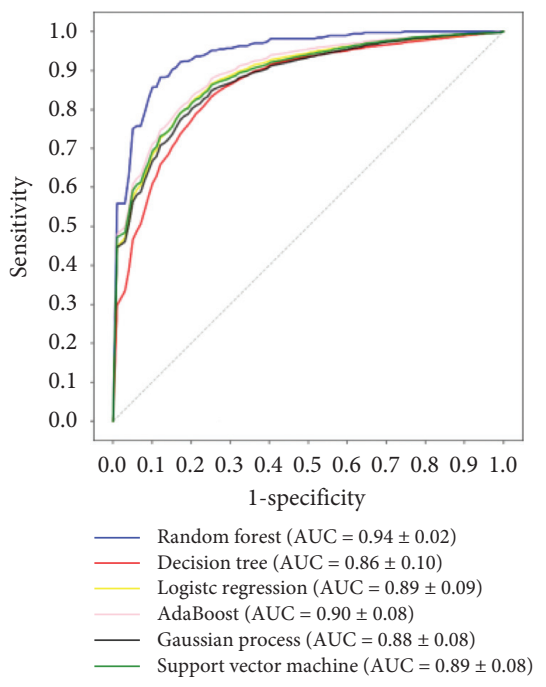

(a)

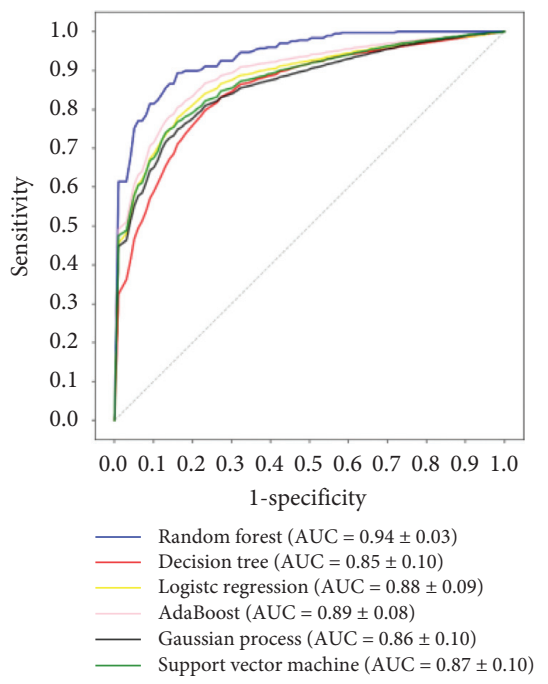

(d)

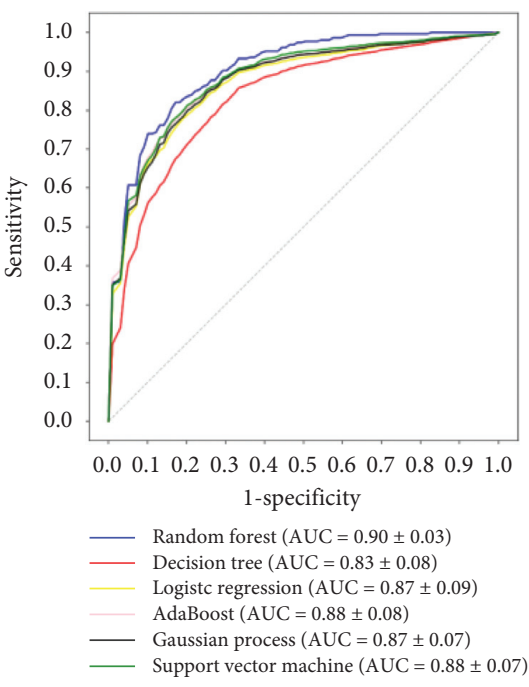

(b)

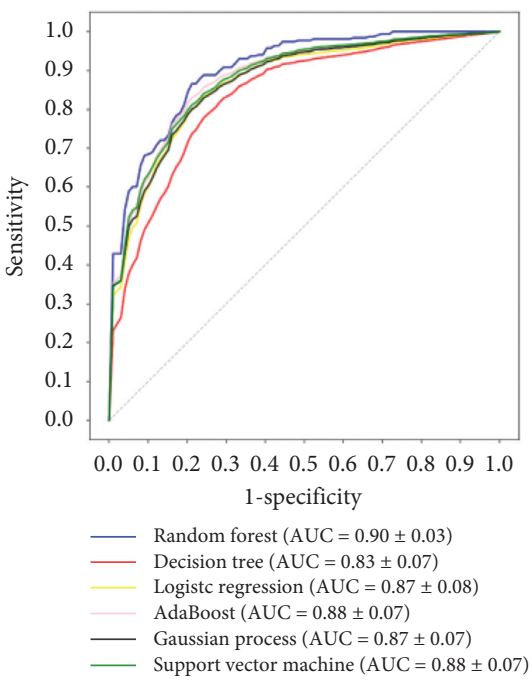

(e)

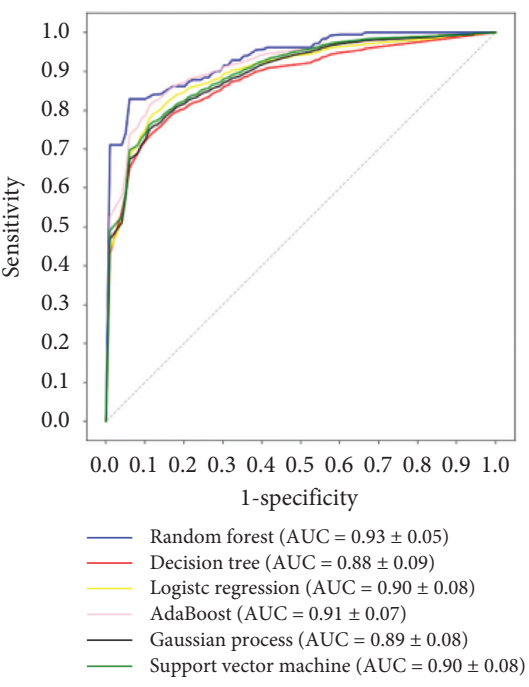

(c)

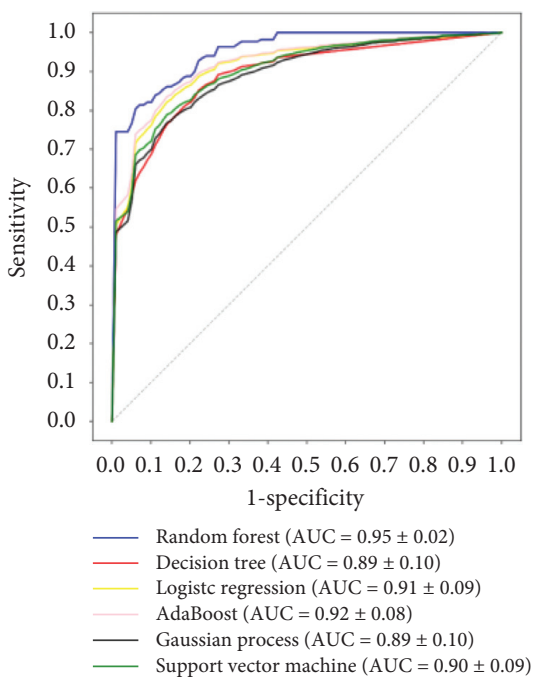

(f)

FIGURE 3: Performance of different machine learning modeling methods in 6 feature groups. (a)-(c) ROC curves of the HCR models based on T1CE, T1WI, and T2WI data, respectively. (d)-(f) ROC curves of the HCR + DLR models based on T1CE, T1WI, and T2WI data, respectively.

comparison of the performance of the multimodality and single-modality models.

\section{Discussion}

The accurate classification of GBM and single BM lesions is a challenging clinical problem. Here, we provide a radiomic method based on HCR combined with DLR that showed potential for clinical application.

In this study, to overcome the disadvantages of current studies, we used different machine learning methods to preoperatively classify GBM and BM using a series of radiomic features. We evaluated tumor features throughout the tumor area rather than performing a largest 


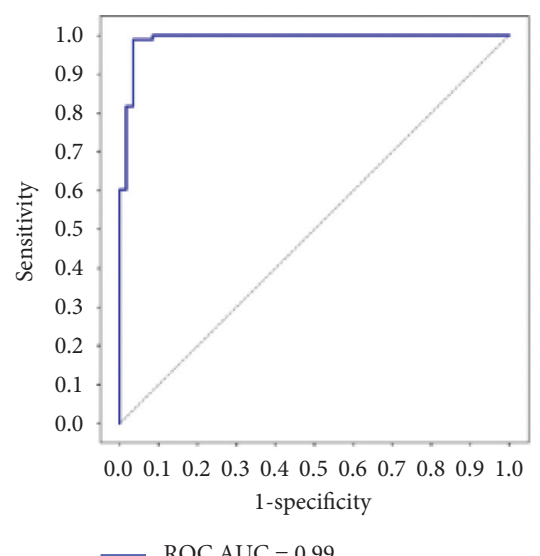

(a)

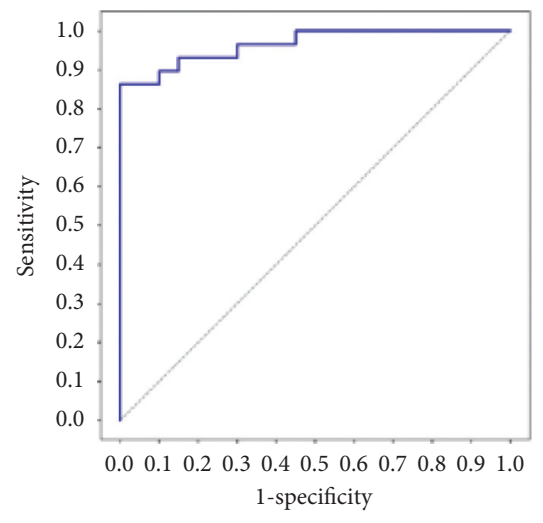

ROC AUC $=0.97$

(d)

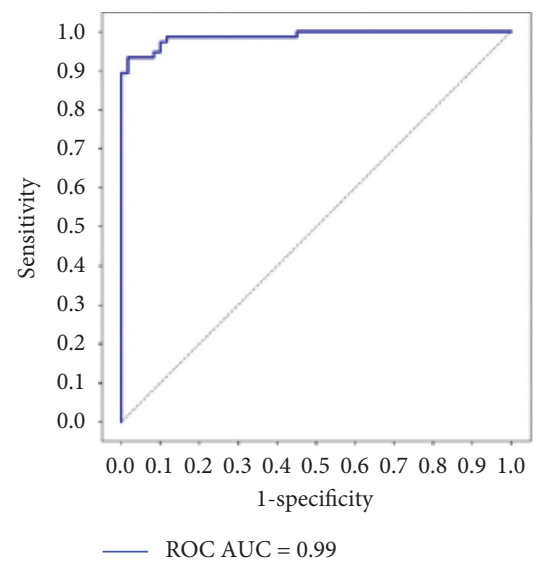

(g)

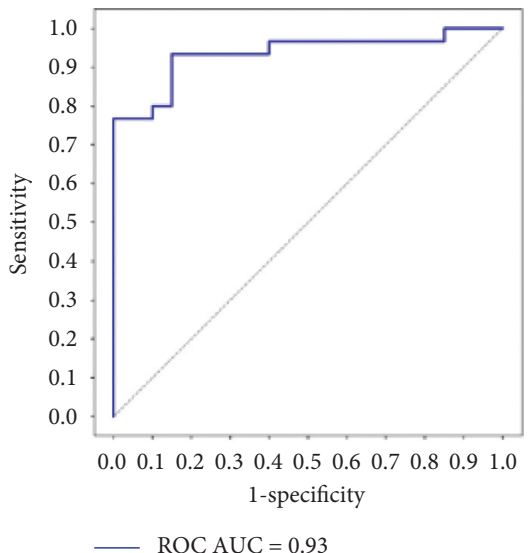

(b)

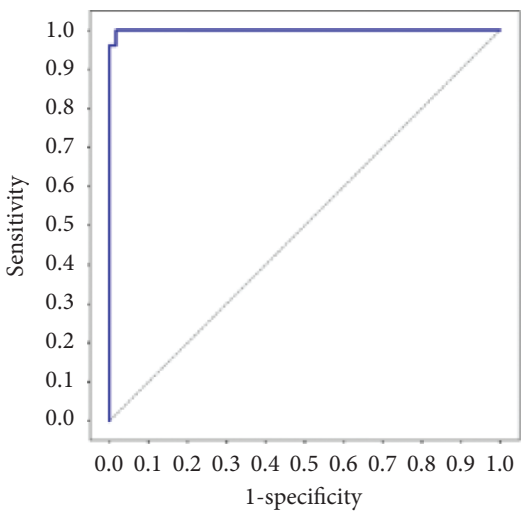

ROC AUC $=1.00$

(e)

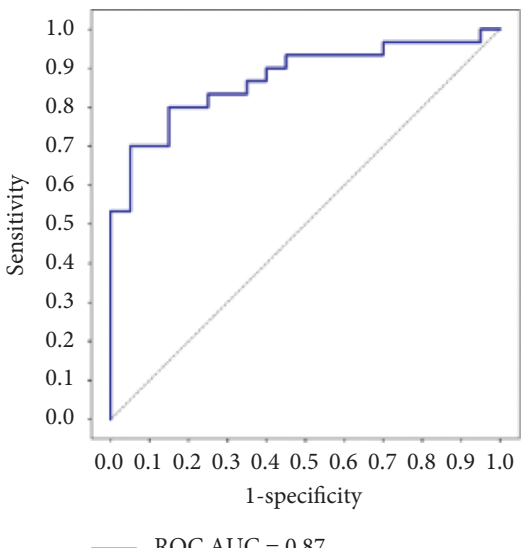

(h)

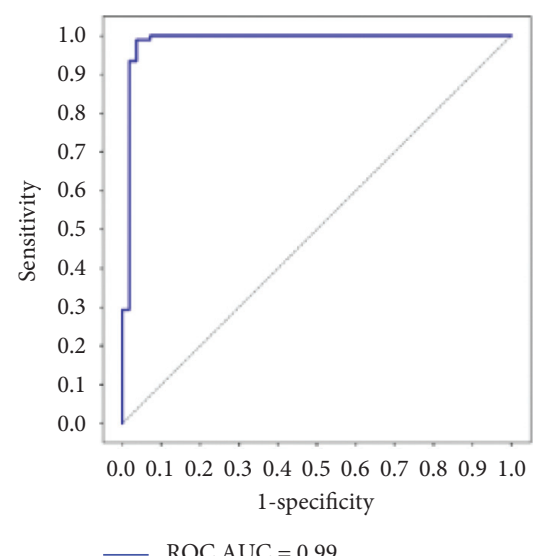

(c)

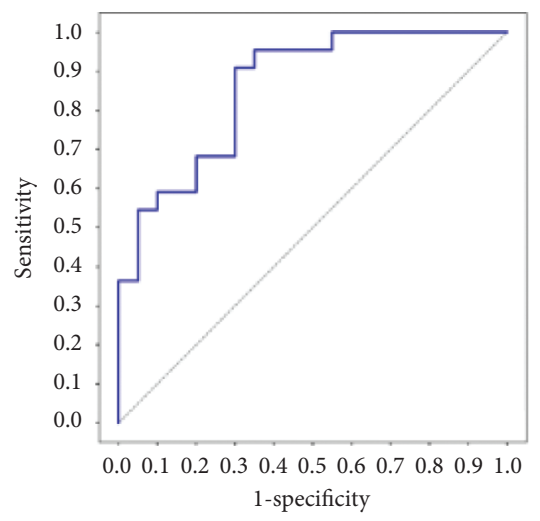

ROC AUC $=0.86$

(f)

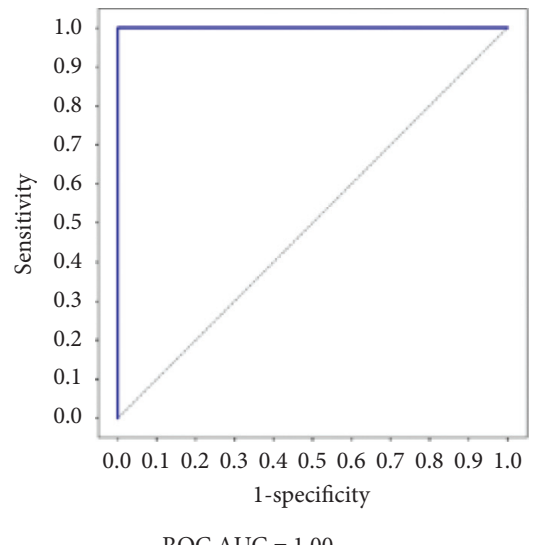

(i)

FIgURE 4: Continued. 


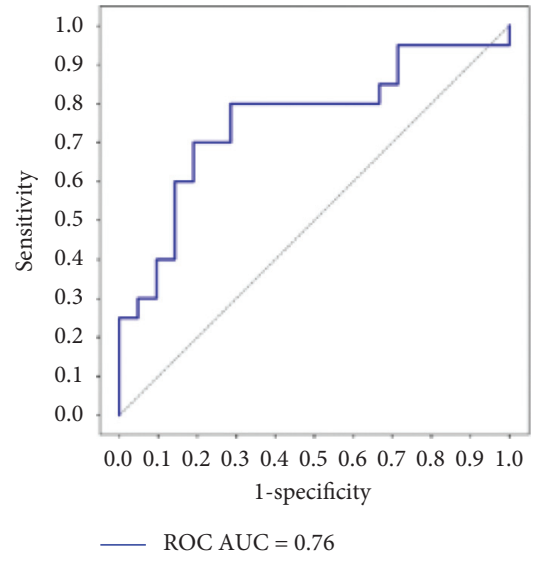

(j)

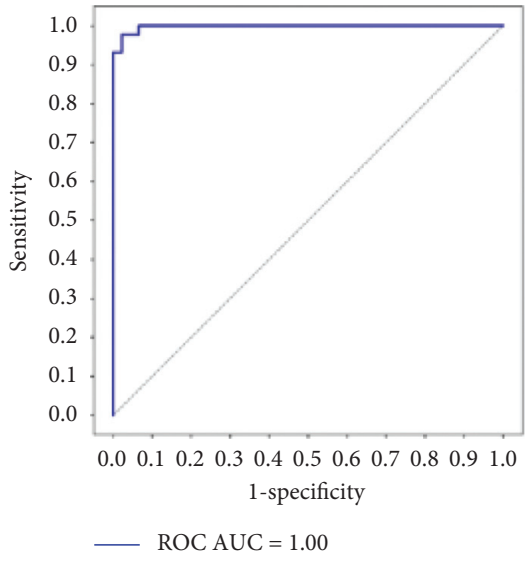

(k)

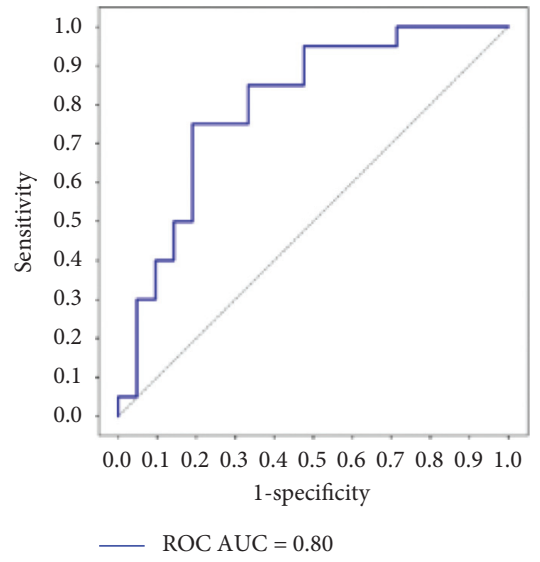

(l)

FIGURE 4: Diagnostic performance of 6 feature groups. (a)-(b) ROC curves of the HCR model based on T1CE data in the training dataset and test dataset, respectively. (c)-(d) HCR + DLR models of T1CE data in the training and test datasets. (e)-(h) HCR and HCR + DLR models of T1WI data in the training dataset and test dataset. (i)-(l) Details of T2WI data.

TABLE 2: The performance comparison of multimodality and single-modality models.

\begin{tabular}{lcccccc}
\hline Models & Training AUC & Cross-validation mean AUC & Test AUC & Accuracy & Sensitivity & Specificity \\
\hline T1CE-HCR & 0.99 & 0.94 & 0.93 & 0.82 & 0.70 \\
T1CE-HCR+DLR & $\mathbf{0 . 9 9}$ & $\mathbf{0 . 9 5}$ & $\mathbf{0 . 9 7}$ & $\mathbf{0 . 8 5}$ & $\mathbf{0 . 8 4}$ \\
T1WI-HCR & 1.00 & 0.90 & 0.86 & 0.71 & 0.75 \\
T1WI-HCR+DLR & 0.99 & 0.91 & 0.87 & 0.76 & 0.75 \\
T2WI-HCR & 1.00 & 0.95 & 0.76 & 0.73 & 0.85 \\
T2WI-HCR+DLR & 1.00 & 0.96 & 0.80 & 0.78 & 0.80 \\
Multimodality-HCR & 1.00 & 0.92 & 0.81 & 0.71 & 0.70 \\
Multimodality-HCR + DLR & 1.00 & 0.96 & 0.84 & 0.75 & 0.73 \\
\hline
\end{tabular}

The bold values represent the highest accuracy and specificity.

cross-sectional analysis, since the analysis of three-dimensional tumor features may provide more diverse internal information than that of two-dimensional features. The majority of these features, such as wavelet, second-order, and some first-order statistical features, cannot be identified and quantified by the human eye, which highlights the advantage of using automatic methods and extracting highorder statistical features to assist in radiological assessment and clinical decision making. Furthermore, we carried out 10 -fold cross-validation and multicenter verification, which enabled us to reduce regional deviation and improve the universality of the approach. The predictive models were built using HCR and DLR analysis. Based on HCR features, six different machine learning algorithms were implemented, including RF, decision tree, logistic regression, AdaBoost, Gaussian processing, and support vector machine. The RF classifier showed the best performance (AUC $=0.93$ ). The combined radiomic model, based on the optimal HCR features and DLR features, showed the best overall performance, with an AUC of 0.99 in the training set and 0.97 in the test set. However, we should note that the differences in performance among the different models were not large enough to select one optimal model, specifically considering that the investigated models seemed to perform quite comparably and that the variance in AUC might be partially attributed to the small sample size. Therefore, our results can only be regarded to support our hypothesis and need to be verified in future studies.

ResNet was proposed in 2015 as a CNN to solve the problem of deep networks, such as the vanishing gradient [34]. Residual neural networks reduce the complexity by skipping the connection that skips training from a few layers and connects directly to the output. Skipping effectively simplifies the network, using fewer layers in the initial training stages, makes it easier to optimize, and provides additional accuracy from a considerably increased depth, which makes it possible to train up to hundreds or even thousands of layers and still achieve compelling performance. Taking advantage of its image classification capability, ResNet is a powerful potential tool for clinical imaging diagnosis. Some other deep learning methods have also been developed to classify and detect brain tumors [35, 36], all of which showed the clinical potential of artificial intelligence technology.

Generally, the potential of multimodality MRI for application may be better than that of single-modality MRI, but there are some cases in which its performance can be limited. In this study, the simple combination of selected features from images obtained by different MRI modalities may be one cause of limited performance; we know that more heterogeneous data do not necessarily provide more valuable information. Moreover, some studies have shown that 

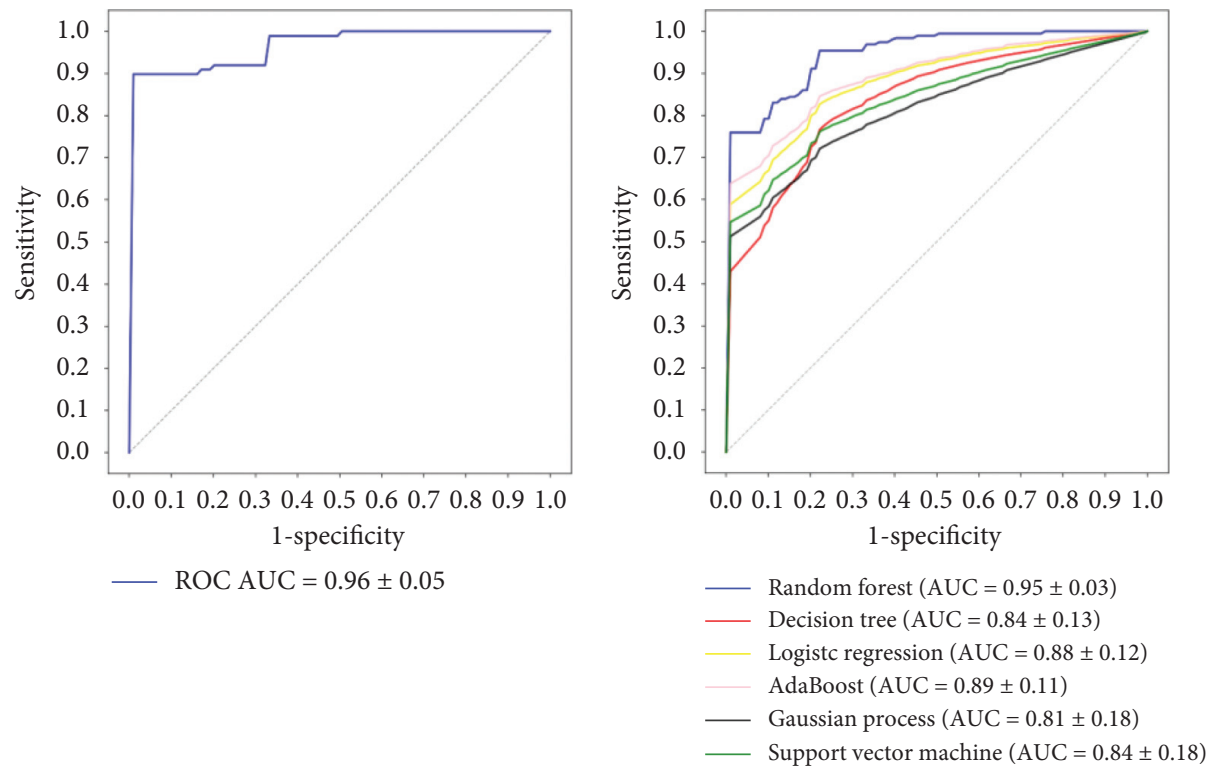

(a)

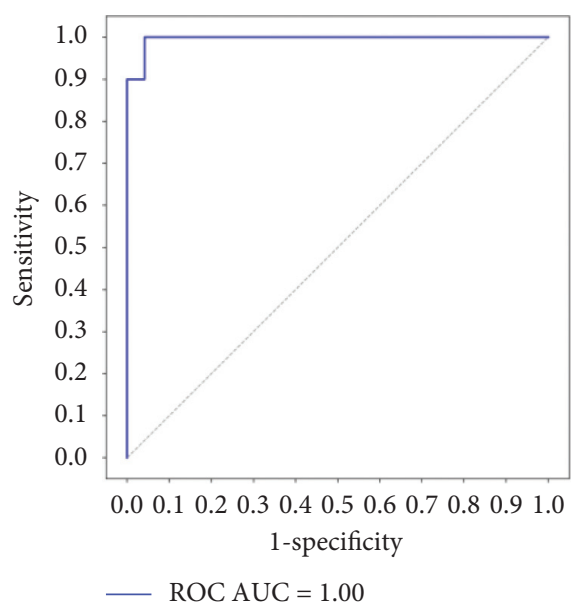

(c)

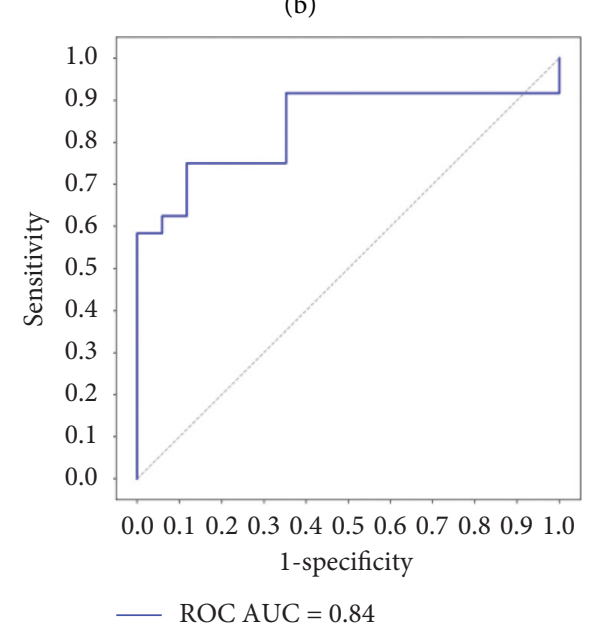

(d)

FIGURE 5: ROC curves of the multimodality radiomic model. (a) Results of 10-fold cross-validation in the training dataset. (b) Comparison of different machine learning models. (c) Final multimodality model in the training dataset. (d) Final multimodality model in the test dataset.

single-modality models may have predictive potential better than or equal to that of multimodality models $[37,38]$. We think that detrimental features usually decrease the overall performance of the model.

There are some limitations to this study that should be addressed. First, this was a retrospective analysis of a small sample. Second, the ratio of BM to GBM lesions and the variety of $\mathrm{BM}$ sources were not representative of the general population, which could exhibit different characteristics. Thus, a larger prospective study is needed for verification. Third, in this study, a manual method was used to segment ROIs. Although manual segmentation is usually better than automatic segmentation and two researchers participated in the segmentation process, segmentation errors still occur. Fourth, we did not compare the performance of the machine learning model with that of a human reader.

\section{Conclusions}

In conclusion, our study suggests a radiomic machine learning method for distinguishing BM from GBM lesions preoperatively with favorable predictive accuracy and stability. In addition, this combination could yield valuable insight into tumor progression, which could also facilitate the implementation of a personalized approach in tumor management. However, the results of our study need to be used with caution; we only validated the feasibility of applying HCR and DLR for the accurate preoperative classification of GBM and solitary BM lesions.

\section{Abbreviations}

MRI: $\quad$ Magnetic resonance imaging

ROIs: $\quad$ Regions of interest 
T1WI/T2WI: T1/T2-weighted images

T1CE: $\quad$ Contrast-enhanced T1

HCR: $\quad$ Handcrafted radiomics

DLR: $\quad$ Deep learning-based radiomics

ROC: $\quad$ Receiver operating characteristic

AUC: $\quad$ Area under curve

RF: $\quad$ Random forest

ResNet: $\quad$ Residual network

LoG: $\quad$ Laplacian of Gaussian.

\section{Data Availability}

The data used to support the findings of this study are available from the corresponding author upon request. The main codes are provided in https://github.com/JZK00/ Radiomics-Features-Extractor. The radiomics data are provided in https://github.com/JZK00/BM-GBM.

\section{Conflicts of Interest}

The authors declare that they have no conflicts of interest.

\section{Acknowledgments}

This work was supported by National Multidisciplinary Cooperative Diagnosis and Treatment Capacity Building Project for Major Diseases of China (lung cancer), major scientific research issues of China Medical Education Association in 2020 (2020KTS010), and Beijing Xisike Clinical Oncology Research Foundation-Heng Rui Oncology Research Foundation (Y-HR2019-0185).

\section{Supplementary Materials}

Supplementary Materials 1: the details of original and selected features of each feature group. Supplementary Materials 2: the robust performance of the training dataset using 10-fold cross-validation. (Supplementary Materials)

\section{References}

[1] B. D. Fox, V. J. Cheung, A. J. Patel, D. Suki, and G. Rao, "Epidemiology of metastatic brain tumors," Neurosurgery Clinics of North America, vol. 22, no. 1, pp. 1-6, 2011.

[2] Q. T. Ostrom, G. Cioffi, H. Gittleman et al., "CBTRUS statistical report: primary brain and other central nervous system tumors diagnosed in the United States in 2012-2016," NeuroOncology, vol. 21, 2019.

[3] C. Adamson, O. O. Kanu, A. I. Mehta et al., "Glioblastoma multiforme: a review of where we have been and where we are going," Expert Opinion on Investigational Drugs, vol. 18, no. 8, pp. 1061-1083, 2009.

[4] J. T. Sundström, H. Minn, K. K. Lertola, and E. Nordman, "Prognosis of patients treated for intracranial metastases with whole-brain irradiation," Annals of Medicine, vol. 30, no. 3, pp. 296-299, 1998.

[5] R. Stupp, W. P. Mason, M. J. van den Bent et al., "Radiotherapy plus concomitant and adjuvant temozolomide for glioblastoma," New England Journal of Medicine, vol. 352, no. 10, pp. 987-996, 2005.
[6] R. R. Lonser, A. O. Vortmeyer, J. A. Butman et al., "Edema is a precursor to central nervous system peritumoral cyst formation," Annals of Neurology, vol. 58, no. 3, pp. 392-399, 2005.

[7] J. G. Bull, D. E. Saunders, and C. A. Clark, "Discrimination of paediatric brain tumours using apparent diffusion coefficient histograms," European Radiology, vol. 22, no. 2, pp. 447-457, 2012.

[8] S. Cha, J. M. Lupo, M.-H. Chen et al., "Differentiation of glioblastoma multiforme and single brain metastasis by peak height and percentage of signal intensity recovery derived from dynamic susceptibility-weighted contrast-enhanced perfusion MR imaging," American Journal of Neuroradiology, vol. 28 , no. 6 , pp. $1078-1084,2007$

[9] F. C. Maluf, L. M. DeAngelis, J. J. Raizer, and L. E. Abrey, "High-grade gliomas in patients with prior systemic malignancies," Cancer, vol. 94, no. 12, pp. 3219-3224, 2002.

[10] W. Hassaneen, N. B. Levine, D. Suki et al., "Multiple craniotomies in the management of multifocal and multicentric glioblastoma," Journal of Neurosurgery, vol. 114, no. 3, pp. 576-584, 2011.

[11] G. Yang, T. L. Jones, T. R. Barrick, and F. A. Howe, "Discrimination between glioblastoma multiforme and solitary metastasis using morphological features derived from thep: qtensor decomposition of diffusion tensor imaging," NMR in Biomedicine, vol. 27, no. 9, pp. 1103-1111, 2014.

[12] T. J. D. Byrnes, T. R. Barrick, B. A. Bell, and C. A. Clark, "Diffusion tensor imaging discriminates between glioblastoma and cerebral metastases in vivo," NMR in Biomedicine, vol. 24, no. 1, pp. 54-60, 2011.

[13] M. Law, S. Cha, E. A. Knopp, G. Johnson, J. Arnett, and A. W. Litt, "High-grade gliomas and solitary metastases: differentiation by using perfusion and proton spectroscopic MR imaging," Radiology, vol. 222, no. 3, pp. 715-721, 2002.

[14] E. I. Zacharaki, S. Wang, S. Chawla et al., "Classification of brain tumor type and grade using MRI texture and shape in a machine learning scheme," Magnetic Resonance in Medicine, vol. 62, no. 6, pp. 1609-1618, 2009.

[15] I. Tsougos, P. Svolos, E. Kousi et al., "Differentiation of glioblastoma multiforme from metastatic brain tumor using proton magnetic resonance spectroscopy, diffusion and perfusion metrics at 3 T," Cancer Imaging, vol. 12, no. 3, pp. 423-436, 2012.

[16] G. Fan, B. Sun, Z. Wu, Q. Guo, and Y. Guo, "In vivo singlevoxel proton MR spectroscopy in the differentiation of highgrade gliomas and solitary metastases," Clinical Radiology, vol. 59, no. 1, pp. 77-85, 2004.

[17] K. Askaner, A. Rydelius, S. Engelholm et al., "Differentiation between glioblastomas and brain metastases and regarding their primary site of malignancy using dynamic susceptibility contrast MRI at 3T," Journal of Neuroradiology, vol. 46, no. 6, pp. 367-372, 2019.

[18] E. J. Lee, K. terBrugge, D. Mikulis et al., "Diagnostic value of peritumoral minimum apparent diffusion coefficient for differentiation of glioblastoma multiforme from solitary metastatic lesions," American Journal of Roentgenology, vol. 196, no. 1, pp. 71-76, 2011.

[19] B. Pollo, "Pathological classification of brain tumors," The Quarterly Journal of Nuclear Medicine and Molecular Imaging : Official Publication of the Italian Association of Nuclear Medicine (AIMN) [and] the International Association of Radiopharmacology (IAR), [and] Section of the Society, vol. 56, no. 2, pp. 103-111, 2012.

[20] C. Chen, X. Ou, J. Wang, W. Guo, and X. Ma, "Radiomicsbased machine learning in differentiation between 
glioblastoma and metastatic brain tumors," Frontiers in Oncology, vol. 9, p. 806, 2019.

[21] M. D. Kuo and N. Jamshidi, "Behind the numbers: decoding molecular phenotypes with radiogenomics-guiding principles and technical considerations," Radiology, vol. 270, no. 2, pp. 320-325, 2014.

[22] P. Lambin, R. T. H. Leijenaar, T. M. Deist et al., "Radiomics: the bridge between medical imaging and personalized medicine," Nature Reviews Clinical Oncology, vol. 14, no. 12, pp. 749-762, 2017.

[23] R. J. Gillies, P. E. Kinahan, and H. Hricak, "Radiomics: images are more than pictures, they are data," Radiology, vol. 278, no. 2, pp. 563-577, 2016.

[24] C. Yang, Z.-K. Jiang, L.-H. Liu, and M.-S. Zeng, "Pretreatment $\mathrm{ADC}$ image-based random forest classifier for identifying resistant rectal adenocarcinoma to neoadjuvant chemoradiotherapy," International Journal of Colorectal Disease, vol. 35, no. 1, pp. 101-107, 2020.

[25] Y. Huang, Z. Liu, L. He et al., "Radiomics Signature: a potential biomarker for the prediction of disease-free survival in early-Stage (I or II) Non-small cell Lung cancer," Radiology, vol. 281 , no. 3 , pp. $947-957,2016$.

[26] H. J. W. L. Aerts, E. R. Velazquez, R. T. H. Leijenaar et al., "Erratum: corrigendum: Decoding tumour phenotype by noninvasive imaging using a quantitative radiomics approach," Nature Communications, vol. 5, no. 1, p. 4644, 2014.

[27] J.-H. Kim, E. S. Ko, Y. Lim et al., "Breast cancer heterogeneity: MR imaging texture analysis and survival outcomes," Radiology, vol. 282, no. 3, pp. 665-675, 2017.

[28] O. Gevaert, L. A. Mitchell, A. S. Achrol et al., "Glioblastoma multiforme: exploratory radiogenomic analysis by using quantitative image features," Radiology, vol. 273 , no. 1 , pp. 168-174, 2014.

[29] S. Wang, S. J. Kim, H. Poptani et al., "Diagnostic utility of diffusion tensor imaging in differentiating glioblastomas from brain metastases," American Journal of Neuroradiology, vol. 35, no. 5, pp. 928-934, 2014.

[30] C. Liang, Y. Li, and J. Luo, "A novel method to detect functional microRNA regulatory modules by bicliques merging," IEEE/ACM Transactions on Computational Biology and Bioinformatics, vol. 13, no. 3, pp. 549-556, 2016.

[31] J. J. M. van Griethuysen, A. Fedorov, C. Parmar et al., "Computational radiomics system to decode the radiographic phenotype," Cancer Research, vol. 77, no. 21, pp. e104-e107, 2017.

[32] M. B. Kursa and W. R. Rudnicki, "Feature selection with theBorutaPackage," Journal of Statistical Software, vol. 36, no. 11, pp. 1-13, 2010.

[33] M. B. Kursa, A. Jankowski, and W. R. Rudnicki, "Boruta - a system for feature selection," Fundamenta Informaticae, vol. 101, no. 4, pp. 271-285, 2010.

[34] S. Ren, K. He, R. Girshick, X. Zhang, and J. Sun, "Object detection networks on convolutional feature maps," IEEE Transactions on Pattern Analysis and Machine Intelligence, vol. 39, no. 7, pp. 1476-1481, 2017.

[35] M. Soltaninejad, G. Yang, T. Lambrou et al., "Supervised learning based multimodal MRI brain tumour segmentation using texture features from supervoxels," Computer Methods and Programs in Biomedicine, vol. 157, pp. 69-84, 2018.

[36] T. L. Jones, T. J. Byrnes, G. Yang, F. A. Howe, B. A. Bell, and T. R. Barrick, "Brain tumor classification using the diffusion tensor image segmentation (D-SEG) technique," NeuroOncology, vol. 17, no. 3, pp. 466-476, 2015.
[37] Y. Cui, X. Yang, Z. Shi et al., "Radiomics analysis of multiparametric MRI for prediction of pathological complete response to neoadjuvant chemoradiotherapy in locally advanced rectal cancer," European Radiology, vol. 29, no. 3, pp. 1211-1220, 2019.

[38] X. Zhou, Y. Yi, Z. Liu et al., "Radiomics-based pretherapeutic prediction of non-response to neoadjuvant therapy in locally advanced rectal cancer," Annals of Surgical Oncology, vol. 26, no. 6, pp. 1676-1684, 2019. 